\title{
NOTES
}

\section{Cross-effects between Mechanical and Electric Fields in Liquid Crystalline Solution of $\operatorname{Poly}(\gamma$-benzyl L-glutamate)}

\author{
Toshinori IMAI, Tomonori MizunOYa, Kazuyuki HiRaOKA, Takuhei NOSE ${ }^{\dagger}$, \\ Munehiro DATE, and Yoshiko Uematsu \\ Department of Applied Chemistry, Tokyo Institute of Polytechnics, 1583 Iiyama, Atsugi 243-0297, Japan
}

(Received October 20, 2003; Accepted December 15, 2003)

KEY WORDS Poly( $\gamma$-benzyl L-glutamate) / Liquid Crystal / Shear Deformation / Electric Field /
Cross-effect /

Poly( $\gamma$-benzyl L-glutamate) (PBLG) forms a liquid crystal (LC) in a helicogenic organic solvents above a certain concentration. ${ }^{1,2}$ The cholesteric liquid crystalline texture formed by PBLG has been shown the norm due to the optical activity of the PBLG molecule, and nematics under some particular conditions are rather exceptional. ${ }^{3-6}$ Since a PBLG molecule in helical conformation has a large permanent dipole moment along the helical axis, ${ }^{7}$ alignments of dipoles in the cholesteric texture and properties in electric field are very interesting. Application of strong electric field exceeding the threshold value is expected to align the rod-like molecules in the direction of electric field, resulting in a transitional change from cholesteric to nematic texture. In fact, it is the case. ${ }^{8} \mathrm{On}$ the other hand, the mechanical field, i.e., the shear deformation can also bring about the alignment of molecular axes. ${ }^{9}$ Therefore the coupling between electric and mechanical fields through molecular orientation is expected to occur appreciably. To see this coupling, we have investigated effects of electric field on mechanical properties and effects of shear deformation on electric properties, i.e., the cross-effect, for PBLG solution in helicogenic solvent, 1,1,2,2-tetrachlorothane. We have found large cross-effects between electric current and dynamic viscosity with elasticity, which will be reported here.

\section{EXPERIMENTAL}

\section{Materials and Samples}

Poly ( $\gamma$-benzyl-L-glutamate) (PBLG) was synthesized by the $N$-carboxyanhydride method in chloroform with triethylamine as initiator, and subsequently precipitated in hexane. The crude PBLG was repeatedly precipitated by cooling a dilute solution of the polymer in mixed solvent of 1,1,2,2-tetrachloroethane
(TCE) and hexane. The viscosity-average molecular weight was $6 \times 10^{4}$. TCE was a product of Wako Pure Chemical Industries, Ltd. and used as received. Concentration of sample solution was fixed at $0.30 \mathrm{~g} /$ $\mathrm{mL}$. The concentration range of isotropic-to-cholesteric transition around room temperature for the TCE solution of the present PBLG sample is located at concentrations lower than $30 \mathrm{wt} \%$, i.e., the concentrations of the A point and the B point are approximately $14 \mathrm{wt} \%$ and $19 \mathrm{wt} \%$, respectively, so that the sample solution used here is in a cholesteric liquid crystalline state under no electric field.

\section{Electric and rheological Measurements}

The apparatus was a RheoStress of Haak Co., RS75 , which is a stress-control type rheometer, modified to be able to apply an electric field, and is illustrated in Figure 1. Sample solution was loaded between rotators of parallel plate type with $35 \mathrm{~mm}$ diameter. The plates were separated by $0.2 \mathrm{~mm}$, between which electric field can be applied perpendicular to the plates, using a generator, Multifunctional Synthesizer 1920A produced by NF Electronic Instruments Co. The electric current was measured by a home-made electric current-to-field translator, being monitored and memorized by an oscilloscope (Textronix, TDS3014) and a personal computer. A sample cover and cotton fiber wetted with TCE prevent solvent evaporation. Temperature was controlled at $25^{\circ} \mathrm{C}$ within $\pm 0.1{ }^{\circ} \mathrm{C}$.

The complex modulus of dynamic viscoelasticity was measured as a function of frequency at constant stress amplitudes under applying static electric field ranging from 0 to $750 \mathrm{~V} / \mathrm{cm}$. Electric current at a fixed static electric field was measured under dynamic mechanical stress as a function of time after starting to apply the shear stress. Dynamic viscoelastisity meas-

${ }^{\dagger}$ To whom correspondence should be addressed (Tel/Fax: 046-242-9524, E-mail: nose@chem.t-kougei.ac.jp). 


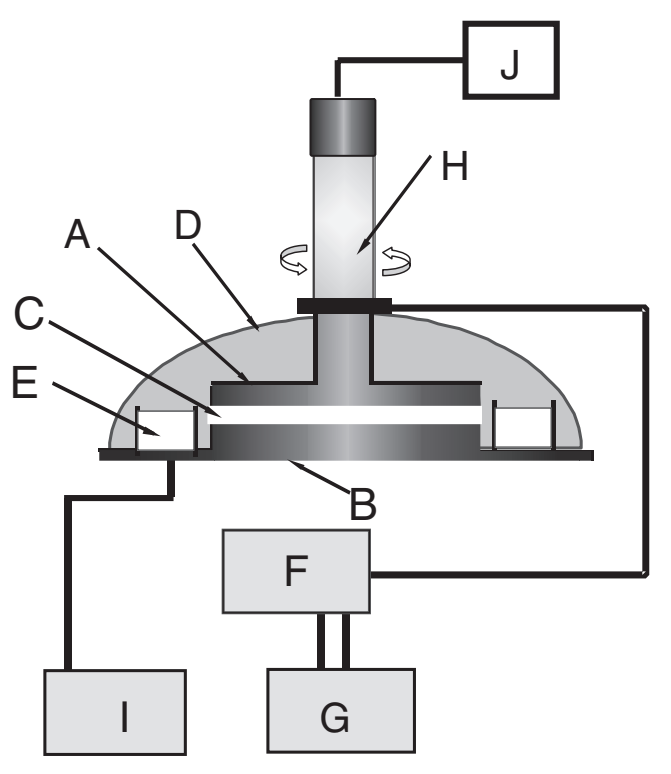

Figure 1. Schematic illustration of an apparatus for electrorheorogical measurements. A, B: parallel plates serving as electrodes, too; C: sample; D: cover for preventing solvent evaporation; E: cotton wetted with solvent; F: serving both as an electric-pressure generator and an electric-current to electric-field converter; G: oscilloscope; H: ceramic rod; I: temperature controller; and $\mathrm{J}$ : computer controlling viscoelastic measurements.

urements were performed at shear strains of the order of $100 \%$, and the non-linearity was not so strong, which enabled us to analyze data by the conventional way using the complex modulus.

Direct current observed was approximately proportional to applied electric field, and of the order of $10 \mu \mathrm{A}$ under $100 \mathrm{~V} / \mathrm{cm}$, so that the electric conductivity was of the order of $10^{7} \Omega^{-1} \mathrm{~cm}^{-1}$ for $9.62 \mathrm{~cm}^{2}$ of plate area. It should be noted here that the electric conductivity is substantially none for pure solvent, TCE, so that the conductivity of the solution comes from the presence of the solute, PBLG in solution. Therefore direct electric current has to be due to some charges generated form PBLG.

\section{RESULTS AND DISCUSSION}

\section{Changes in Rheological Properties by Electric Field}

Figure 2 illustrates changes of the dynamic viscosity, $\eta^{\prime}$, the storage modulus, $G^{\prime}$, and the loss modulus, $G^{\prime \prime}$, with the lapse of time, when the electric field is increasingly applied at the rate of $50 \mathrm{~V} / \mathrm{cm}$ per minute up to be $750 \mathrm{~V} / \mathrm{cm}$ of electric field, $E$, and then switched off to be zero at the time of $900 \mathrm{~s}$. At lower voltages, no effects of electric field are observed on the mechanical properties, where the solution exhibits behavior of a viscous fluid with no elasticity (undetectable $G^{\prime}$ ), and shows no change of viscosity with increasing voltage. Around $E=300 \mathrm{~V} / \mathrm{cm}$, the viscosity starts to increase with increasing $E$, and further increase of $E$ brings about the elasticity. That is, at about $500 \mathrm{~V} / \mathrm{cm}$, the $G^{\prime}$ shows up to be detectable, and increases with increasing voltage.

The threshold voltage for appearance of appreciable effect of electric field is around $300-400 \mathrm{~V} / \mathrm{cm}$, and almost corresponds to the critical electric field for the divergence of cholesteric pitch, that is, the change from cholesteric to homeotoropic nematics. The critical voltages for the $0.30 \mathrm{~g} / \mathrm{mL}$ solutions are, for example, $470 \mathrm{~V} / \mathrm{cm}$ and $505 \mathrm{~V} / \mathrm{cm}$ for PBLG with molecular weight $\left(M_{\mathrm{W}}\right)=214,000$ in TCE, and PBLG with $M_{\mathrm{W}}=56,000$ in dioxane, respectively. ${ }^{10}$

The increment of viscosity with appearance of elasticity is supposed to need the orientation of aligned PBLG rod-like molecules perpendicular to shear deformation. Electric field to maintain the orientation is needed as well to see the mechanical response.

As also seen in Figure 2, the sudden stop of applying electric field brings about a sharp drop of dynamic viscosity, and instantaneous disappearance of the storage modulus. However, some aftereffect in viscosity is observed for about $100 \mathrm{~s}$. This may imply that the relaxation (or retardation) for the response of molecular motion is relatively quick, but has a tail of the order of $100 \mathrm{~s}$.

To have more detailed information on this, are shown in Figure 3 the dynamic viscosity changes under electric field with lapse of time when the electric field starts to apply at the same time as, or $10 \mathrm{~min}$ before, the shear stress starts to be applied. In the case of simultaneous start of application of electric and mechanical fields, we observe a quick increase in dynamic viscosity, followed by a slow retardation of viscosity increment. Molecular rotation effective to the viscoelasticity occurs in relatively short time, and it takes more time, of the order of $100 \mathrm{~s}$, to complete

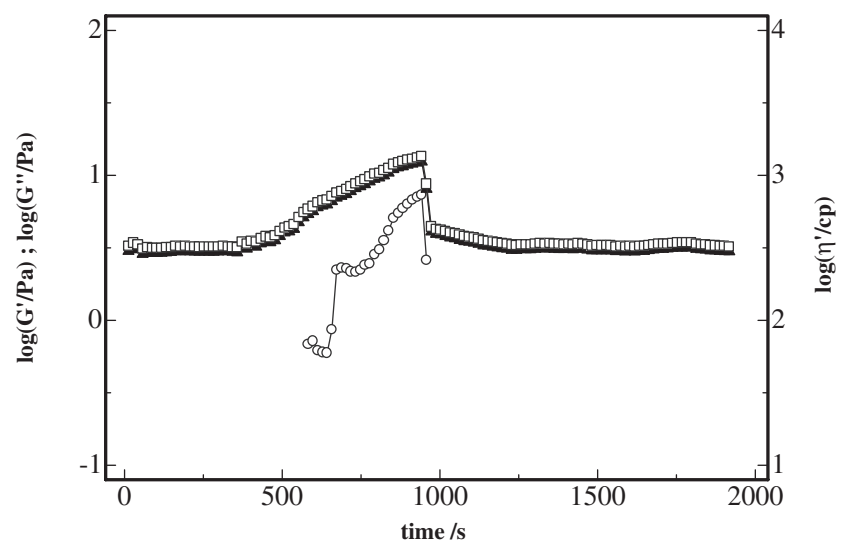

Figure 2. Changes in dynamic viscosity, $\eta^{\prime}$, storage shear modulus, $G^{\prime}$, and loss shear modulus, $G^{\prime \prime}$, measured at $\omega=9.24 \mathrm{rad} / \mathrm{s}$ with stress amplitude $\sigma=17.8 \mathrm{~Pa}$ under stepwise increase of voltage of static electric field at the rate of $50 \mathrm{~V} / \mathrm{cm}$ per min, followed by quit of applying electric field at $900 \mathrm{~s}$

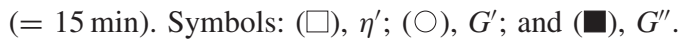



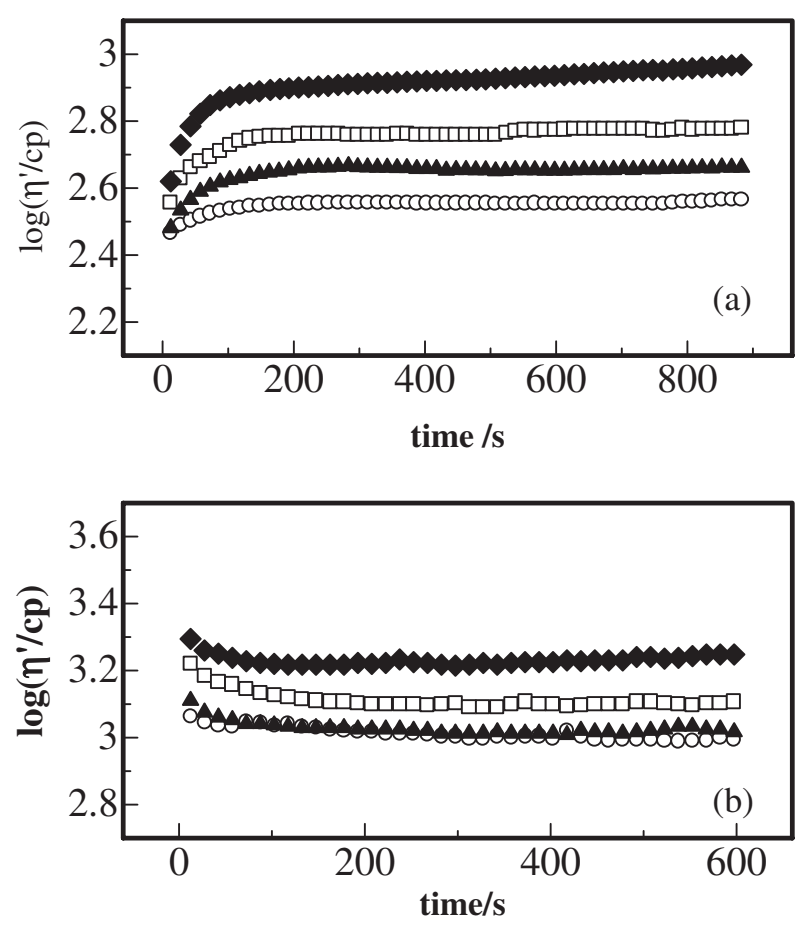

Figure 3. Changes in dynamic viscosity, $\eta^{\prime}$, measured at $\omega=9.24 \mathrm{rad} / \mathrm{s}$ with $\sigma=17.8 \mathrm{~Pa}$ when the static electric field starts to be applied (a) at the same time as, and (b) $10 \mathrm{~min}$ before, the start of shear deformation. Applied electric fields: $(\bigcirc), 100 \mathrm{~V} /$ cm; $(\mathbf{\Delta}), 200 \mathrm{~V} / \mathrm{cm} ;(\square), 300 \mathrm{~V} / \mathrm{cm}$; and $(\diamond), 400 \mathrm{~V} / \mathrm{cm}$.

the steady alignment. On the other hand, in the case of advanced application of electric field, a shallow decay of the viscosity in about $100 \mathrm{~s}$ is observed only. In this case, the molecular orientation by electric field is supposed to have substantially been finished before application of mechanical field, the viscosity decay may reflect the time needed to reach the fine alignment under mechanical field. It should be noted that the solution under electric field does not reach the same steady state in both cases as yet in the present time scale, which is easily recognized by different electric currents even at long times (Figure 3). So far we have no definite idea for this finding, but one possibility is that it may be partly due to a very slow relaxation of molecular alignments at the surface of sample-loaded plates.

These findings of relaxational aspects mentioned above seem to consistently show that the molecular rotation primarily needed for the cross-effect takes place in relatively short time, followed by the fine adjustment according to the conditions of electric and mechanical fields, which takes a longer time of the order of $100 \mathrm{~s}$.

\section{Electric Current Induced by Mechanical Deformation}

Figure 4 illustrates time-variation of electric current after giving sinusoidal shear deformation under a fixed electric field for various voltages, where the
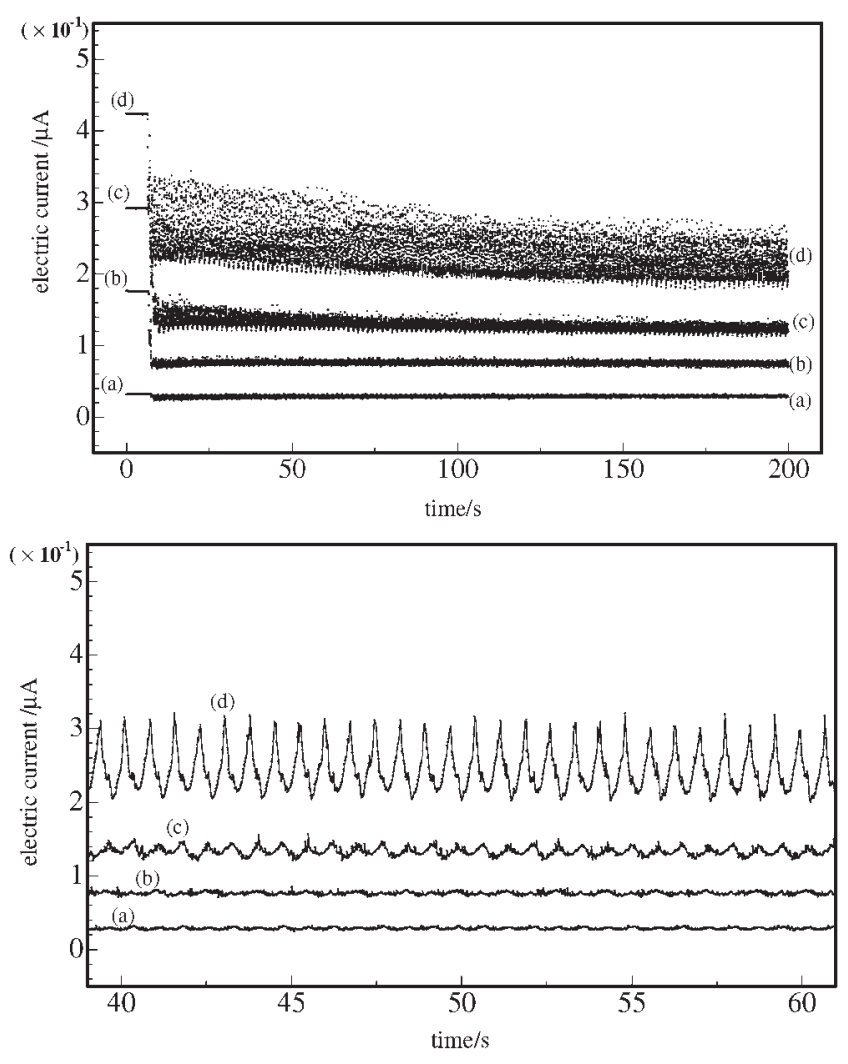

Figure 4. Electric-current response as a function of time after applying dynamic shear stress $(\omega=4.28 \mathrm{rad} / \mathrm{s}$; stress amplitude $\sigma=11.9 \mathrm{~Pa}$ ) under various strengths of electric field. (a) $100 \mathrm{~V} /$ $\mathrm{cm}$; (b) $200 \mathrm{~V} / \mathrm{cm}$; (c) $300 \mathrm{~V} / \mathrm{cm}$; and (d) $400 \mathrm{~V} / \mathrm{cm}$. Bars at $t=0$ indicate electric currents before applying shear deformation. The lower figure represents the data in an enlarged time scale for the limited time range.

electric field starts to be applied $5 \mathrm{~min}$ before applying the shear. The time recorded in the horizontal axis of the figure is shifted by about $10 \mathrm{~s}$ from the starting time of shear to measure and show the electric current right before starting the share deformation. At the highest voltage of static electric field, $400 \mathrm{~V} / \mathrm{cm}$, the electric current quickly drop by starting the dynamic deformation, followed by vibrational changes synchronized with the dynamic stress. The response of current as a function of time is not a simple sinusoidal vibration, but has steep tops and round bottoms alternatively, seeming to reduce the current value being proportional to the absolute value of sinusoidal stress variation with phase difference. Namely, the electric current seems to depend on the amplitude of strain irrespective of direction of shear deformation. Such response of current to mechanical deformation cannot be detected at low voltages, and becomes detectable with increasing voltage, but still subtle at $300 \mathrm{~V} / \mathrm{cm}$, although the initial drop of the current is appreciable even at low voltages. The strength of electric field inducing the vibrational response needs to be over a threshold value. The appearance of dynamical current corresponds to the changes in viscoelasticity 


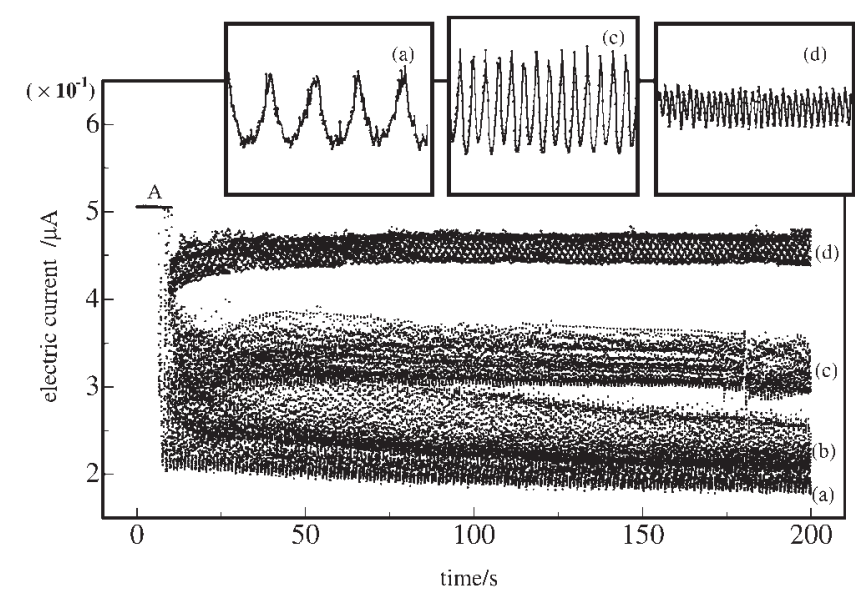

Figure 5. Electric-current response as a function of time after applying dynamic shear stress (stress amplitude $\sigma=11.9 \mathrm{~Pa}$ ) under electric field of $400 \mathrm{~V} / \mathrm{cm}$ for various frequencies. Stress frequency $\omega$ : (a), $2.91 \mathrm{rad} / \mathrm{s}$; (b), $4.28 \mathrm{rad} / \mathrm{s}$; (c), $9.24 \mathrm{rad} / \mathrm{s}$; and (d), $19.9 \mathrm{rad} / \mathrm{s}$. The bar (A) at $t=0$ indicates the current before applying shear deformation. Insets represent the data in an enlarged time scale for the limited time range of about $40-45 \mathrm{~s}$.

by static electric field shown in Figure 2. This critical voltage can again be pointed out to correspond to that for the homeotropic nematic molecular orientation by static electric field.

The above findings suggest that the molecular alignment is a necessary condition for the dynamic current, and that the dynamic current is responding to the deviation of the molecular orientation from the direction of electric field, which is perpendicular to the shear-applying direction.

Figure 5 shows stress-frequency $(\omega)$ dependency of electric current. The amplitude of vibrational response is substantially independent of $\omega$, while the initial drop of the current decreases with increasing frequency. Seeing the results of Figures 4 and 5 more carefully, one can also notice a tail of the relaxational decay of steady electric current on which the vibrational current is laid. The decay time is of the order of $50 \mathrm{~s}$, which is the same order of, but slightly shorter than, the relaxation (or retardation) time of dynamic viscosity decay (or retardation) after releasing (or applying) the electric field, as seen in Figures 2 and 3.

The change of steady current with a large drop at starting of dynamic shear deformation tells us something about the origin of electric current under static electric field, which is not observed for pure solvent but for PBLG solution. Free charges generated from PBLG by electric field may depend on the frequency.
This is consistent with the quick response, i.e., the initial drop, to the imposition of mechanical field. The tails observed in both of time-dependencies of viscosity and electric current are presumed to come from subtle adjustments to more stable molecular alignments.

Finally we have to point out that the cross-effects discovered here can be observed rather in the limited ranges of electric field strength and shear stress. If the electric field is too high, then more complex behavior in molecular orientation are observed, exhibiting complex domain pattern in liquid crystalline texture. The higher stress, which implies the larger strain, leads to the reduction of vibrational response probably because of disturbance of layer flow. The stimulation of a low level cannot give the cross-effect, which is essentially a non-linear phenomenon.

In conclusion, we have found a large cross-effect between electric field and mechanical deformation for liquid crystalline solution of PBLG in TCE. The increase in dynamic viscosity accompanying with the appearance of elasticity is induced by electric field applied over the critical strength. Corresponding to this, sinusoidal dynamic shear stress brings about vibrational electric current above the critical strength of electric field. The electric current is presumed to be reduced responding to the absolute strain of dynamic deformation, leading to the vibrational current. The cross-effect is suggested to be related to the orientation of aligned rod-like PBLG molecules, which is maintained by electric field and perturbed by mechanical field.

\section{REFERENCES}

1. C. Robinson, Trans. Faraday Soc., 52, 571 (1956).

2. C. Robinson, J. C. Ward, and R. B. Beevers, Discuss. Faraday Soc., 25, 29 (1958).

3. S. Sobajima, J. Phys. Soc. Jpn., 23, 1070 (1967).

4. E. T. Samulski and A. V. Tobolsky, Macromolecules, 1, 555 (1968).

5. C. Robinson, Tetrahedron, 13, 219 (1961).

6. R. W. Duke, D. B. Dupre, W. A. Hines, and E. T. Samulsky, J. Am. Chem. Soc., 98, 3094 (1976).

7. A. Wada, J. Chem. Phys., 30, 328 (1959).

8. R. W. Duke and D. B. DuPre, Macromolecules, 7, 374 (1974).

9. G. Kiss and R. S. Porter, J. Polym. Sci., Polym. Phys. Ed., 18, 361 (1980); Mol. Cryst. Liq. Cryst. 60, 267 (1980).

10. Unpublished data. 